\title{
Hydrogen-enhanced Surface Reactivity of X80 Pipeline Steel observed by Scanning Electrochemical Microscopy

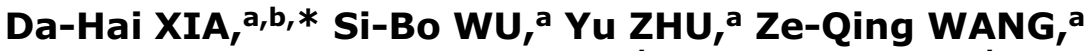 \\ Ying-Hao SUN, ${ }^{a}$ Ren-Kang ZHU, ${ }^{b}$ and Jing-Li LUO ${ }^{b, *}$
}

\author{
a Tianjin Key Laboratory of Composite and Functional Materials, School of Materials Science and Engineering, \\ Tianjin University, Tianjin 300072, People's Republic of China public of China \\ b Department of Chemical and Materials Engineering, University of Alberta, \\ Edmonton, Alberta, Canada, T6G 2V4
}

* Corresponding authors: dahaixia@tju.edu.cn, jingli.luo@ualberta.ca

\begin{abstract}
Effect of hydrogen on the surface reactivity of X80 pipeline steel was studied by scanning electrochemical microscopy (SECM), electrochemical impedance spectroscopy (EIS) and secondary ion mass spectroscopy (SIMS). Hydrogen can enhance the reactivity of passive film formed on X80 pipeline steel, reduce the corrosion resistance and thickness of this passive layer, which is due to an increase in hydroxide in the passive layer.
\end{abstract}

(C) The Electrochemical Society of Japan, All rights reserved.

Keywords : Hydrogen, Surface Reactivity, SECM, EIS

\section{Introduction}

Hydrogen damage of pipeline steels is a severe problem that refers to the degradation of mechanical properties of pipeline steels caused by the presence of, or interaction with, hydrogen. ${ }^{1}$ The reduction of hydrogen ions at the metal/solution interface is a source of atomic hydrogen. Hence, corrosion reactions, the application of cathodic protection and electroplating, etc., are major sources for ingress of hydrogen into the pipeline steels. ${ }^{2}$ During these hydrogen-generating processes, some of the reduced hydrogen may absorb onto the metal surface and diffuse into the metals. The presence of hydrogen in metals has a significant impact on the mechanical and electrochemical behaviours of metals. For example, increasing hydrogen content of steels results in the low stress fracture and pitting susceptibility. Dissolved hydrogen in metals was proven to increase the dissolution rates of alloys in both the passive and transpassive regions by hindering chromium enrichment in surface films ${ }^{3}$ and to induce stress corrosion cracking and pitting corrosion. ${ }^{4,5}$ Incorporation of hydrogen into the passive film would alter the semiconductivity of the passive layer on AISI 310 stainless steel. ${ }^{6}$

In order to fully understand the hydrogen effect on the properties of passive film, various techniques are needed, including surface analysis methods like secondary ion mass spectroscopy (SIMS), ${ }^{7,8}$ X-ray photoelectron spectroscopy (XPS), ${ }^{7}$ as well as electrochemical methods like electrochemical impedance spectroscopy (EIS), ${ }^{9,10}$ electrochemical noise. ${ }^{11}$ Previous works mainly focused on hydrogen effects on the electrochemical behaviour and physicochemical property of the surface film, but limited investigations have been conducted focusing on the surface reactivity. In recent years, scanning electrochemical microscopy (SECM) has been developed for a wide range of applications in corrosion science. ${ }^{12,13}$ An advantage of SECM is its capability to probe charge transfer occurring nonuniformly at interfaces. ${ }^{14-17}$ Zhu et al. investigated the impact of tensile and compressive stress on reactivity of the Alloy $800 \mathrm{C}$-ring sample using SECM and found that both tension and compression increased the localized surface reactivity, indicating corrosion susceptibility of Alloy 800 under stress in thiosulfate-contained chemistries. ${ }^{18}$

In this work, effect of hydrogen on surface reactivity of pipeline steel was studied by SECM, and the hydrogen effects on film composition and thickness were also investigated.

\section{Experimental}

\subsection{Materia}

The testing material used in this work was cut from a hot-rolled X80 steel plate, with a chemical composition (wt\%): $0.07 \mathrm{C}$, $0.216 \mathrm{Si}, 1.8 \mathrm{Mn}, \quad 0.182 \mathrm{Mo}, 0.168 \mathrm{Ni}, 0.266 \mathrm{Cr}, 0.221 \mathrm{Cu}$, $0.105 \mathrm{Nb}, \quad 0.026 \mathrm{Al}, \quad 0.013 \mathrm{Ti}, \quad 0.001 \mathrm{~V}, \quad 0.003 \mathrm{~N}, \quad 0.0137 \mathrm{P}$, $0.0009 \mathrm{~S}$ and $\mathrm{Fe}$ balance. Prior to each experiment, the surface of each specimen was polished with wet silicon carbide papers in the sequence of $320,600,800,1200$, and 3000 grit, and then mechanically polished by a polishing cloth, rinsed copiously with deionized water and cleaned with ethanol and acetone, then dried in desiccator for $24 \mathrm{~h}$. The exposed area was $3.118 \mathrm{~cm}^{2}$.

\subsection{Electrochemical methods}

Figure 1 shows the flow chart of sample preparation procedure. All electrochemical measurements were conducted at room temperature in aerated solutions. EIS measurements were performed at corrosion potential by using an EG\&G PAR model 273 potentiostat. A three-electrode cell was used, using X80 as the working electrode (WE), saturated calomel electrode (SCE) as the reference electrode and platinum electrode as the counter electrode (CE). The applied signals had a $10 \mathrm{mV}$ amplitude with frequencies ranging from $100 \mathrm{kHz}$ to $0.01 \mathrm{~Hz}$. The surface reactivity was mapped by SECM. The electrolyte contained $0.9 \mathrm{mM}$ ferrocenylmethanol $(99 \%$, Aldrich) as the redox mediator and $0.12 \mathrm{M} \mathrm{Na}_{2} \mathrm{~S}+1.0 \mathrm{M} \mathrm{NaOH}$ (Fisher, reagent grade, $>99 \%$ ) as the supporting electrolyte. Deionized water (Milli-Q, Millipore, $18.2 \mathrm{M} \Omega \mathrm{cm}$ resistivity) was used to prepare all solutions. The solution during all measurements was exposed to the air. Before the electrochemical measurement, the $\mathrm{X} 80$ specimen was polarized at $-1 \mathrm{~V}_{\mathrm{SCE}}$ for $1 \mathrm{~h}$ to reduce the oxide layer.

SECM was worked at the constant-distance mode, meaning that the distance between prove and surface of specimen is a constant value during the scanning process. For SECM experiments, an epoxy-mounted specimen was sandwiched between two blocks of PTFE to form a cell. This cell was mounted on the SECM stage (CHInstruments, USA). The potentials in this paper are presented in SCE scale. For hydrogen-charged specimens, the X80 pipeline steel was polarized in $0.12 \mathrm{M} \mathrm{Na}_{2} \mathrm{~S}+1.0 \mathrm{M} \mathrm{NaOH}$ solution at $-1.3 \mathrm{~V}_{\mathrm{SCE}}$ 


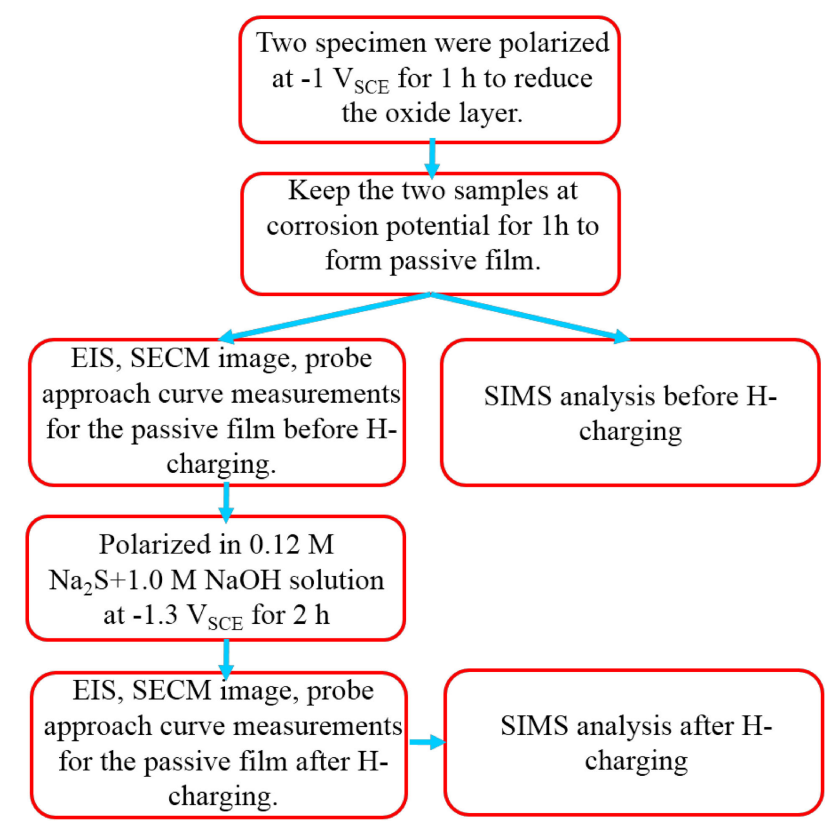

Figure 1. (Color online) The flow chart of sample preparation procedure.

for $2 \mathrm{~h}$. An ultramicroelectrode (UME) with a radius of $10 \mu \mathrm{m}$ and ratio of glass diameter of 5 was used as the SECM probe. An SCE was used as the RE and a Pt wire was the CE. The probe was biased at a potential of $0.4 \mathrm{~V}_{\mathrm{SCE}}$ until steady-state current was obtained, and then the probe was driven to the specimen surface in order to accurately determine the gap distance between the probe and the specimen. When the UME current $\left(i_{T}\right)$ reached the prescribed value, the tip stopped automatically. For an insulating surface, tip stopped automatically when $i_{T}$ decreased to half of the steady-state current; for an conductive surface, tip stopped automatically when $\mathrm{i}_{\mathrm{T}}$ increased to 2 times of the steady-state current. The normalized current $\left(\mathrm{I}_{\mathrm{T}}\right.$, the measured tip current divided by the tip current measured in bulk solution) vs. the normalized distance ( $\mathrm{L}$, the distance between the tip and specimen divided by the radius of UME) was plotted as the probe approach curve (PAC). The real gap distance between the tip and the specimen was estimated by comparing experimental PACs with simulated/theoretical PACs, as shown in the previous work. ${ }^{14,19-22}$ The UME gap distance was maintained at constant and scanned above the specimen to obtain the SECM images of the X80 surface. The passive film exhibits semiconductor properties, and it was considered to be an inactive film. The model we used to fit PACs is developed by Renkang Zhu and Zhifeng Ding. ${ }^{13}$

\subsection{Time-of-flight secondary ion mass spectrometry}

Time-of-flight secondary ion mass spectrometry (ToF SIMS) analysis was carried out using a ToF SIMS IV instrument (ION-ToF $\mathrm{GmbH})$. The information depth of ToF SIMS analysis was limited to the top 1 to 20 monolayers. Ions from mass 1 (hydrogen) to $\sim 9000 \mathrm{amu}$ (for cluster ions) were detected with resolutions ranging from $1 \mathrm{ppb}$ to $\mathrm{ppm}$ level of concentrations, depending on the element. In the current work, the analysis source used was $\mathrm{Ga}^{+}$, operating at $15 \mathrm{kV}$; the sputtering source was $\mathrm{Cs}^{+}$, operating at $1 \mathrm{kV}$. The samples were immersed in the solution until the SIMS test to minimize atmospheric oxidation. Before the test, specimens were cleaned with distilled water. A reference X80 specimen was used SIMS calibration by sputtering the sample for 600 s and measuring the sputtering depth as $64.14 \mathrm{~nm}$, so sputtering rates of alloying elements could be determined and hence accurate contents of alloying elements could be determined with the depth as well as accurate depth. The sputtering rate was $0.1069 \mathrm{~nm} / \mathrm{s}$.

\subsection{Scanning electron microscopy and Energy Dispersive Spectroscopy}

Scanning electron microscopy (SEM) analyses were carried out on the specimen surface using a field-emission scanning electron microscope (FE-SEM, Hitachi, Japan) equipped with Genesis XM2 Energy Dispersive Spectroscopy (EDS).

\section{Results and Discussion}

Figure 2(a) and (b) show surface morphology of X80 pipeline steel after grinding. Inclusions in dark colour were observed to distribute randomly in the steel matrix. Figure 2(c) shows compositions of 5 inclusions typically existing in X80 steel. Points 1 was an $\mathrm{Al}$, $\mathrm{Ca}$-enriched inclusion which is possibly in the form of $\mathrm{CaO}-\mathrm{Al}_{2} \mathrm{O}_{3}$. Inclusion 2,3,4,5 were an $\mathrm{Al}$ oxide inclusion, with small amount of $\mathrm{Mg}$ and $\mathrm{Ca}$. Point 6 was located in the substrate region where there was no inclusion, therefore $\mathrm{Ca}, \mathrm{S}, \mathrm{Mg}, \mathrm{Al}$, Ti were not detectable. The sizes of inclusions were at a micrometer scale.

Figure 3 shows the schematic illustration of the SECM experiments and the PAC of the passive film formed on X80 pipeline steel before $\mathrm{H}$-charging. The following reaction occurred on the probe when it biased at $0.4 \mathrm{~V}_{\mathrm{SCE}}$ to obtain the steady-state current:

$$
\mathrm{Fc} \rightarrow \mathrm{Fc}^{+}+\mathrm{e}
$$

This current was much higher than the $\mathrm{S}^{2-}$ and $\mathrm{OH}^{-}$oxidation current on the probe, ${ }^{18}$ because the oxidation current of $0.12 \mathrm{M} \mathrm{Na}_{2} \mathrm{~S}$ and $1.0 \mathrm{M} \mathrm{NaOH}$ at $0.4 \mathrm{~V}_{\mathrm{SCE}}$ on Pt electrode is $0.2 \mathrm{nA}$ and $0.01 \mathrm{nA}$ respectively. Therefore, the oxidation current of these two ions was negligible. When the probe was close enough to the conductive surface, the product of the probe, ferroceniummethanol $\left(\mathrm{Fc}^{+}\right)$, diffused to the alloy surface and was reduced:
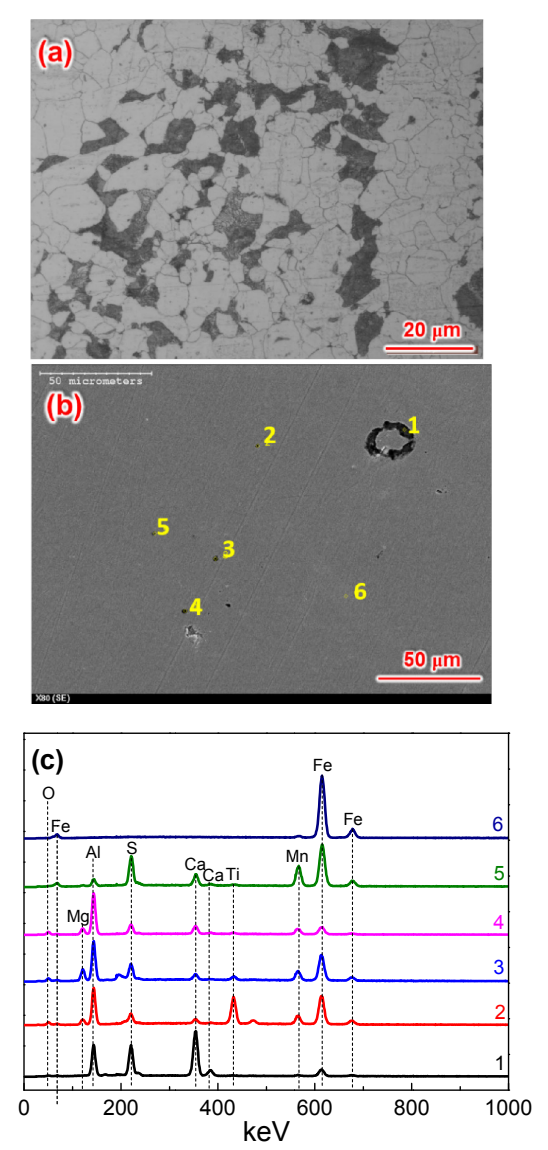

Figure 2. (Color online) (a) microstructure of X80 steel (b) inclusions in X80 steel (c) EDS results of X80 pipeline steel. 
(a)
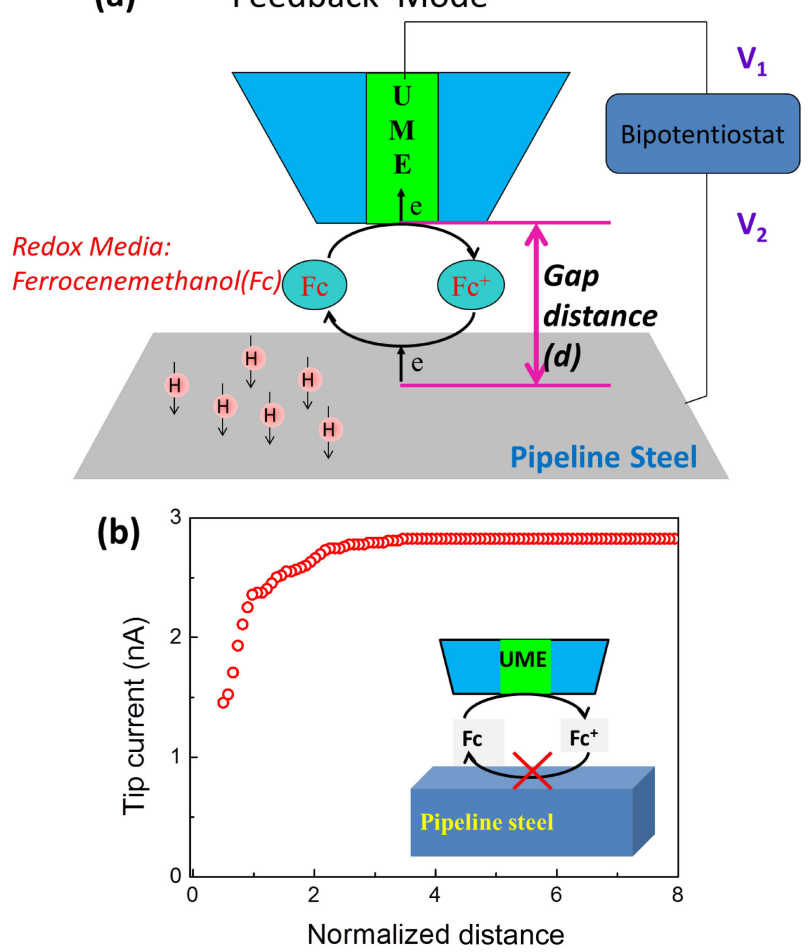

Figure 3. (Color online) (a) Schematic illustration of the SECM experiments (b) the PAC of the passive film formed on X80 pipeline steel before $\mathrm{H}$-charging.

$$
\mathrm{Fc}^{+}+\mathrm{e} \rightarrow \mathrm{Fc}
$$

The reduced $\mathrm{Fc}$ diffused again to the probe surface and contributed to $\mathrm{i}_{\mathrm{T}}$. Rate constant shown in this paper is the rate of this reaction. Figure 3(b) shows the PAC of the passive film formed on X80 pipeline steel before H-charging. The oxide current in bulk solution was about $2.8 \mathrm{nA}$, The tip current can be calculated by the following equation at diffusion control process: $:^{23}$

$$
i_{T, \infty}=4 x n F D c_{0} a
$$

where $x$ is the coefficient related with RG ( $x=5$ in this paper); $n$ the number of electrons transferred in the tip reaction (here, $\mathrm{n}=1$ ); $F$ the Faraday constant; $D$ the diffusion coefficient of species $\mathrm{Fc}$ $\left(D=3.3 \times 10^{-7} \mathrm{~cm}^{2} / \mathrm{s}\right) ;{ }^{20} a$ the tip radius $(a=5 \mu \mathrm{m}) ;$ and $c_{0}$ the concentration of $\mathrm{Fc}$ in the bulk solution $\left(c_{0}=0.9 \mathrm{mM}\right)$. The calculated $i_{T}$ is about $2.87 \mathrm{nA}$, which is equal to $2.8 \mathrm{nA}$ shown in the PAC.

The UME current decreased when the UME was driven close to the $\mathrm{X} 80$ surface. Therefore, a negative feedback was observed on the $\mathrm{X} 80$ surface, indicating that X80 was covered by an oxide layer.

At a constant height of $2.7 \mu \mathrm{m}$ (constant height means constant distance between prove and surface of specimen), the SECM UME was rastered in the $X, Y$ plane above the X80 surface using the closed-loop positioning system, and the tip currents versus the lateral tip positions were recorded to obtain SECM images. Figure 4(a) shows the SECM images of the passive film before $\mathrm{H}$ charging. The tip current range for this image was $0.9-1.3 \mathrm{nA}$. Some spots with a higher current than their surroundings can be observed in this image, which is possibly due to inclusions. As shown in Fig. 2(b), the size of some inclusions in X80 is large and is around $30 \mu \mathrm{m}$ or even larger. Comparatively, the SECM image of the specimen $\mathrm{H}$-charging is shown in Fig. 4(b), and the tip current range for this image was $1.2-2.6 \mathrm{nA}$. It should be noted that, after $\mathrm{H}$ charging, the specimen was kept at corrosion potential for at least $1 \mathrm{~h}$ to form an H-containing passive film. It is believe that if there
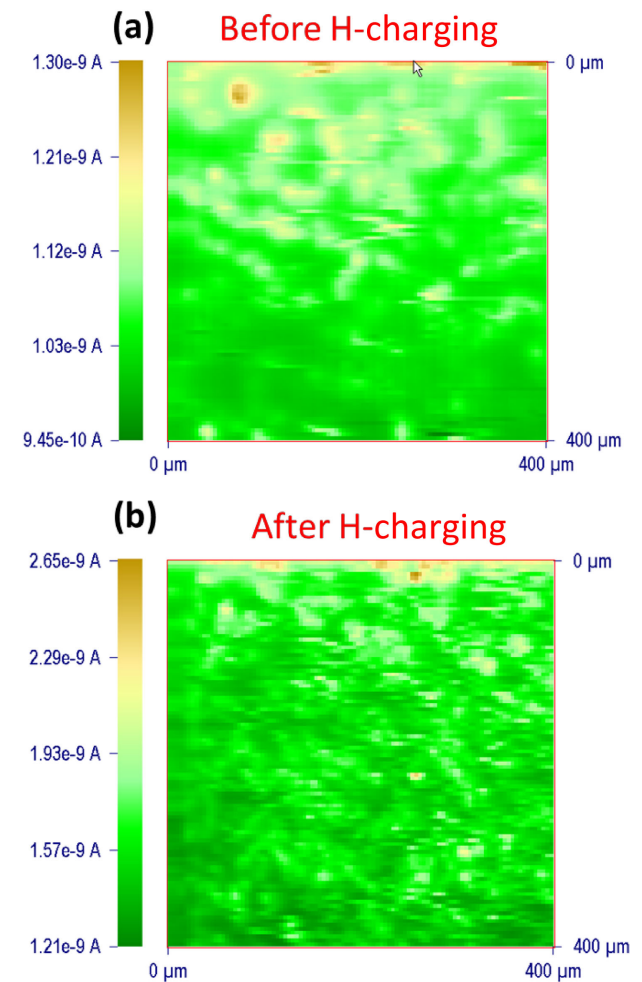

Figure 4. (Color online) SECM images of the passive film (a) before and (b) after H-charging.

was some hydrogen discharged, and the discharging should be done during this one-hour time because the discharging process is fast. Therefore, hydrogen is the main factor that affect the surface reactivity. So the oxidation reaction on the tip would not affected by the discharging of hydrogen. It is evident that the current distribution on the surface alters, mainly due to the change in morphology and composition of the passive film. After hydrogen charging, atomic bonds were weakened, defects were increased, and local surface plasticity was increased.

Figure 5(a) normalized probe approach curves (PACs) toward X80 surface before and after H-charging. The PACs expected for an insulator (black line) and a conductor (grey line) are also plotted for comparison. Superimposed on these plots are the simulated PACs obtained using the model described above. Note that a rate constant of $6.8 \times 10^{-4} \mathrm{~cm} / \mathrm{s}$ before H-charging was determined by fitting, which is distinguishable from an insulator. After H-charging, the rate constant increases to $3.1 \times 10^{-3} \mathrm{~cm} / \mathrm{s}$, which is $\sim 5$ times larger than that before $\mathrm{H}$-charging. PAC results indicate that hydrogen enhances surface reactivity of X80.

EIS was obtained after immersing in solution at corrosion potential for $1 \mathrm{~h}$ for the specimens before and after $\mathrm{H}$-charging, and the results are shown in Fig. 5(b). One time constants format are considered for such a system. This form of impedance is consistent with the occurrence of a charge transfer reaction on the interface and a charge-discharge process in the interfacial capacitance, therefore, the EIS data can be fitted using a charge transfer resistance in parallel with a capacitance Q. The charge transfer resistances of the system are $488 \mathrm{k} \Omega \mathrm{cm}^{2}$ before H-charging which is possibly due to $\mathrm{g}$ and $258 \mathrm{k} \Omega \mathrm{cm}^{2}$ after $\mathrm{H}$-charging. Apparently, an $\mathrm{H}$-charging passive film shows a poorer corrosion resistance than the uncharged passive film.

In order to confirm effect of hydrogen on $\mathrm{O}$ and $-\mathrm{OH}$ content in the passive film, SIMS was performed to analyze film composition and film thickness, with the results shown in Fig. 6. The thickness of the passive film can be well recognized in Fig. 6(b), because O 


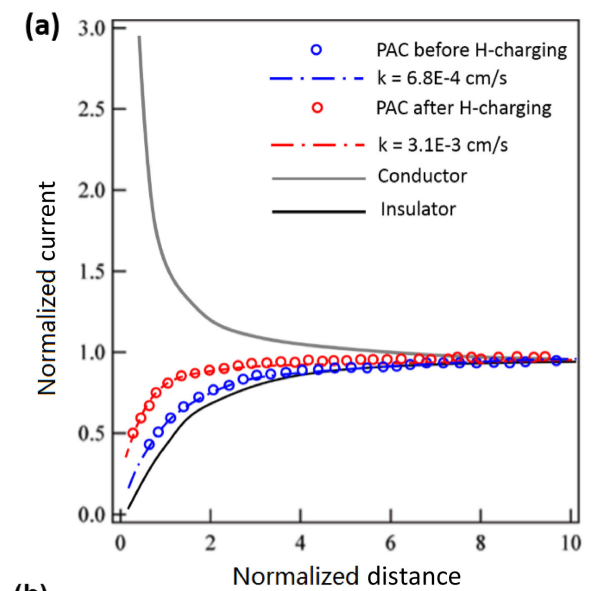

(b)

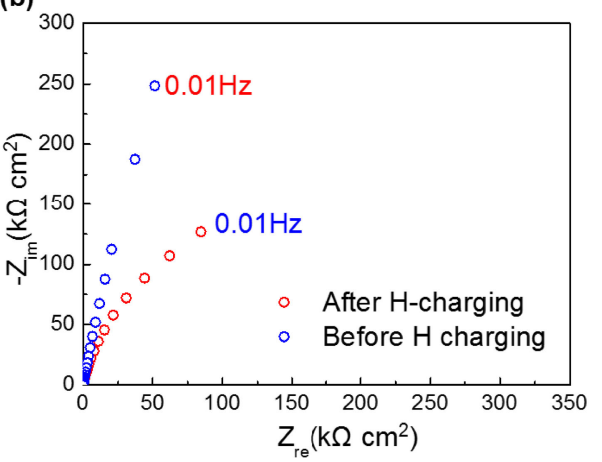

Figure 5. (Color online) (a) Probe approach curves (PACs) toward X80 surface before and after H-charging. The PACs expected for an insulator (black line) and a conductor (grey line) are also plotted for comparison. The tip approach speed was at $1.0 \mu \mathrm{m} / \mathrm{s}$. The corresponding simulated PACs are superposed to the experimental ones. (b) EIS of X80 pipeline steel before and after $\mathrm{H}$-charging.

content is high in the passive film, and is low in the substrate. Before $\mathrm{H}$-charging, the thickness of passive film is about $4 \mathrm{~nm}$, and it lowers to $2 \mathrm{~nm}$ after H-charging. It is generally accepted that film thickness is determined by the film dissolution rate at the film/ solution interface and oxygen vacancy generation rate at the metal/ film interface. A decreased film thickness is possibly due to an increase in film dissolution rate and subsequently increases the transportation rates of oxygen vacancies and metal vacancies in the film. Figure 6 also illustrates that the -OH intensity of an $\mathrm{H}$-charging film is significantly higher than that of the uncharged passive film. The results indicate that hydrogen increases hydroxide content in the passive layer by the reaction (4)

$$
\mathrm{H}^{+}+\mathrm{O}^{2-} \rightarrow \mathrm{OH}^{-}
$$

$\mathrm{X} 80$ is an iron-base alloy, the passive film should be iron oxide or hydroxide. Iron oxide is usually considered as n-type semiconductor which can be attributed to the presence of oxygen vacancies in the passive film. According to the point defect model (PDM), ${ }^{24-26}$ the following reaction occurs at the film/solution interface

$$
\mathrm{V}_{\mathrm{O}}^{\ddot{*}}+2 \mathrm{OH}^{-} \rightarrow \mathrm{O}_{\mathrm{O}}+\mathrm{H}_{2} \mathrm{O}
$$

where $\mathrm{V}_{\mathrm{O}}^{*}$ represents the oxygen vacancy at the film/solution interface, $\mathrm{O}_{\mathrm{O}}$ represents an oxygen ion occupying an oxygen vacancy at the same location. A higher concentration of $\mathrm{H}$ at the film/solution interface for H-charging film would retard Reaction 4 in the forward direction. Hence, the concentration of oxygen vacancies at the interfaces may stay at a higher level at steady state for a hydrogen-charged passive film, which makes the passive film less protective.
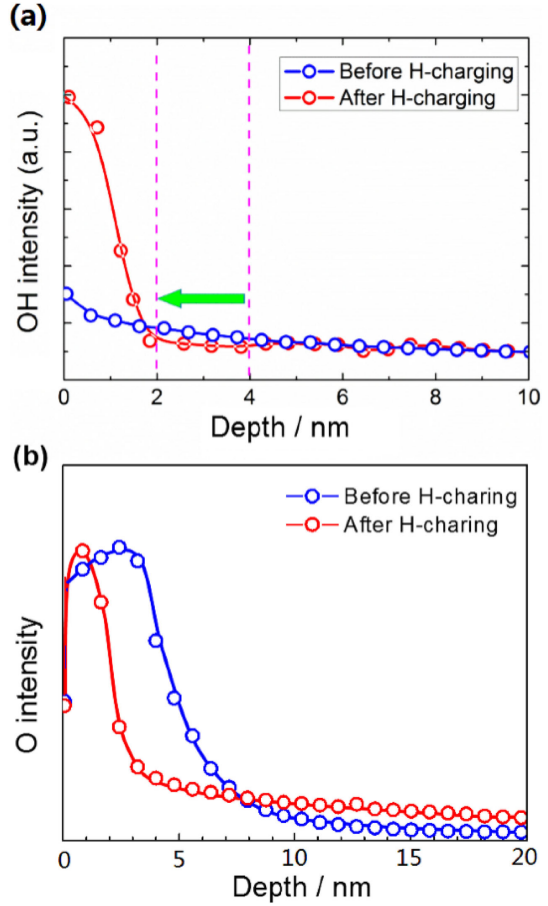

Figure 6. (Color online) Hydroxide and oxygen intensity as function of depth obtained from SIMS (a) $\mathrm{OH}$ (b) $\mathrm{O}$.

\section{Conclusions}

An H-charging passive film shows an increased rate constant than the original passive film, therefore, hydrogen enhances the surface reactivity of X80 pipeline steel. Hydrogen also decreases the corrosion resistance of X80. SIMS result illustrates that hydrogen lowers film thickness and alters film composition by increasing hydroxide content in the passive film.

\section{Acknowledgments}

This work was supported by Supported by the open-ended fund of the Key laboratory of Nuclear Materials and Safety Assessment (Institute of Metal Research, Chinese Academy of Sciences, China) (No. 2016NMSAKF02), National Natural Science Foundation of China (No. 51131007, No. 51371124), the Major State Basic Research Development Program (973 Program) (Granted No. 2014CB046805) and Natural Science Foundation of Tianjin (No. 14JCYBJC17700).

\section{References}

1. Y. Yuan, L. Li, C. Wang, and Y. Zhu, Electrochem. Commun., 12, 1804 (2010).

2. H. B. Xue and Y. F. Cheng, Corros. Sci., 53, 1201 (2011).

3. L. J. Qiao and J. L. Luo, Corrosion, 54, 281 (1998).

4. Q. Yang and J. L. Luo, J. Electrochem. Soc., 148, B29 (2001).

5. M. Z. Yang, J. L. Luo, and B. M. Patchet, Thin Solid Films, 354, 142 (1999).

6. M. Z. Yang, Q. Yang, and J. L. Luo, Corros. Sci., 41, 741 (1999).

7. D. Xia, Y. Sun, and H. Fan, Trans. Tianjin Univ., 21, 554 (2015).

8. D.-H. Xia, Y.-F. Sun, C. Shen, X.-Y. Chen, H.-Q. Fan, and J.-L. Luo, Corros. Sci., 100, 504 (2015).

9. D.-H. Xia and J.-L. Luo, Trans. Tianjin Univ., 21, 234 (2015).

10. D. H. Xia, S. Z. Song, J. H. Wang, H. C. Bi, and Z. W. Han, Trans. Tianjin Univ, 18, 15 (2012).

11. D. H. Xia, S. Z. Song, R. K. Zhu, Y. Behnamian, C. Shen, J. H. Wang, J. L. Luo, Y. C. Lu, and S. Klimas, Electrochim. Acta, 111, 510 (2013).

12. D.-H. Xia, R.-K. Zhu, Y. Behnamian, J.-L. Luo, C.-J. Lin, and S. Klimas, J. Electroanal. Chem., 744, 77 (2015).

13. Z. Gao, Z. Wang, Y. Sun, S. Wu, C. Ma, Y. Zhu, and D. Xia, Trans. Tianjin Univ, accepted for publication (2016). 
14. R. K. Zhu, Z. Q. Qin, J. J. Noel, D. W. Shoesmith, and Z. F. Ding, Anal. Chem., 80, 1437 (2008)

15. K. McKelvey, S. Martin, C. Robinson, and P. R. Unwin, J. Phys. Chem. B, 117, 7878 (2013).

16. K. Fushimi, K. Azumi, and M. Seo, ISIJ Int., 39, 346 (1999).

17. K. Fushimi and M. Seo, ISIJ Int., 42, 1326 (2002).

18. R. K. Zhu and J. L. Luo, Electrochem. Commun., 12, 1752 (2010).

19. R. Zhu and Z. Ding, Can. J. Phys., 83, 1779 (2005).

20. R. Zhu, S. M. Macfie, and Z. Ding, J. Exp. Bot., 56, 2831 (2005)

21. R. K. Zhu, C. Nowierski, Z. F. Ding, J. J. Noel, and D. W. Shoesmith, Chem.
Mater., 19, 2533 (2007).

22. R. K. Zhu, S. B. Xu, G. Podoprygorina, V. Boehmer, S. Mittler, and Z. F. Ding, J. Phys. Chem. C, 112, 15562 (2008).

23. A. J. Bard and M. V. Mirkin, Scanning Electrochemical Microscopy (Ed. M. Dekker), New York, p. 650 (2001).

24. D. D. Macdonald, Electrochim. Acta, 56, 1761 (2011)

25. Y. Zhang, M. Urquidi-Macdonald, G. R. Engelhardt, and D. D. Macdonald, Electrochim. Acta, 69, 1 (2012).

26. F. Mao, S. Sharifi-Asl, J. Yu, and D. D. Macdonald, J. Electrochem. Soc., 161, C254 (2014). 